\title{
Europa ist unsere Antwort auf die neuen globalen Herausforderungen
}

Rainer Funke, MdB, Mitglied des Rechtsausschusses, Berlin

Die Terroranschläge vom 11. September haben die neuen globalen Herausforderungen auf brutale Weise deutlich gemacht. Kein Staat kann einer solchen Herausforderung allein begegnen. Die Mitgliedstaaten der Europäischen Union sind daher mehr denn je in der Pflicht, die Handlungsfähigkeit und Effizienz der EU zu stärken. Nationale Konzepte genügen aber nicht nur für die Terrorbekämpfung nicht mehr. Äußere und innere Sicherheit, ethnische Konflikte und Migrationsströme, Umwelt- und Klimaschutz sowie Probleme der internationalen Kriminalität sind, wenn überhaupt, nur noch in größerem Verbund beherrschbar. Die Blockade in der Reform der europäischen Institutionen muss aufgebrochen werden. Handlungsfähige Organe gehören an die Stelle der Kontaktgruppendiplomatie der großen Mitgliedstaaten der EU.

Europa steht an der Schwelle der endgültigen Überwindung seiner Teilung. Jetzt besteht die historische Chance, ein neues, freies und vereintes Europa zu schaffen. Die vordringlichste Aufgabe für die nächsten Jahre ist daher das Vorantreiben der Ost-Erweiterung der Europäischen Union unter Beteiligung der Bürgerinnen und Bürger. Die europäische Orientierung der Beitrittskandidatenländer darf nicht enttäuscht werden, sonst werden sie sich andere Orientierungen suchen. Dabei kommt dem deutsch-französischen Verhältnis nach wie vor eine Schlüsselrolle zu. Gerade weil beide Länder in der Regel ganz unterschiedliche Interessen und Ausgangspunkte haben, ist ein gemeinsam erzielter Kompromiss, der oft erst nach langen und harten Auseinandersetzungen erzielt wird, so ausgewogen, dass er auch für die anderen Partner akzeptabel erscheint. Nicht in der Übereinstimmung von vornherein, sondern in der Unterschiedlichkeit der Ausgangspositionen liegt das Geheimnis der deutsch-französischen Motorfunktion für Europa. Unverzichtbar sind auf beiden Seiten der Wille zur Kooperation und die Sensibilität, sich gegenseitig nicht zu überfordern, aber auch die Entschlossenheit, die europäische Integration gemeinsam voranzubringen.

Insgesamt muss in der Europapolitik wieder die Frage im Vordergrund stehen, wie die Mitglieder der EU am besten und sachgerechtesten gemeinsame Entscheidungen treffen können, statt darüber nachzudenken, wie Entscheidungen am besten verhindert werden können. Auch die deutsche Politik muss wieder europäisch denken. In den nächsten fünf Jahren stehen in der Europapolitik Aufgaben in vier Kernbereichen auf der Agenda: Europas Handlungsfähigkeit muss sichergestellt werden, die Vereinigung Europas muss vorangebracht werden, Europas Wirtschaft muss gestärkt werden und eine Verfassung für Europas Bürger muss erarbeitet werden. Durch die Erfüllung dieser Aufgaben muss sich die Europäische Union $\mathrm{zu}$ einem starken und fairen Global Player entwickeln.

\section{Die Handlungsfähigkeit Europas sicherstellen}

Die EU ist in den letzten Jahren auf dem Weg zur Sicherung von Effizienz und Handlungsfähigkeit bei gleichzeitiger Finanzierbarkeit auf halbem Wege stecken geblieben. Die bisher begonnenen Reformen zur Vorbereitung der Europäischen Union auf die Erweiterung sowohl in finanzieller als auch in institutioneller Hinsicht weisen zwar in die richtige Richtung, sind aber bei weitem nicht ausreichend. Vor allem hat der Europäische Rat in Nizza in im Dezember 2000 bei der Ausweitung von Mehrheitsentscheidungen im Rat nur marginale Fortschritte erzielt und damit sein hauptsächliches Ziel, die Handlungsfähigkeit der EU zu stärken, verfehlt. Daher ist die EU schon nach den von ihr selbst gesetzten Kriterien nicht im hinreichenden Maße auf ihre Erweiterung vorbereitet. Reformen in den genannten Bereichen sind daher unabdingbar. Sie müssen im Rahmen des europäischen Verfassungsprozesses aufgegriffen und bewältigt werden. Nur wenn ein Durchbruch bei der Ausweitung von Mehrheitsentscheidungen im Rat erreicht wird, kann die Europäische Union ihre Handlungsfähigkeit wiederherstellen und über die Erweiterung hinweg bewahren. Andernfalls wird es europaskeptischen und -feindlichen Kräften in einer sich vergrößernden EU immer leichter gelingen, über das fortbestehende Vetorecht europäische Entscheidungen zu blockieren. Eine Gemeinsame Außen- und Sicherheitspolitik, die diesen Namen verdient, muss schneller als bisher vorangebracht werden. Ziele, Akteure und Instrumente der Gemeinsamen Außen- und Sicherheitspolitik sind endlich klar zu definieren. Dazu müssen zum Beispiel die Aufgaben des Außenkommissars und des Hohen Repräsentanten für die GASP in einer Person zusammengeführt werden. Europa braucht eine Außen- und Sicherheitspolitik mit einer Telefonnummer und aus einem Guss. Die Tatsache, dass die Zeit noch nicht reif ist für ein europäisches Außenministerium oder eine europäische Regierung, ist kein Gegenargument, sondern sollte Ansporn sein, diesen Prozess zu beschleunigen, statt ihm entgegenzuwirken.

Die militärischen Aktionen sind nur ein kleiner Teil der Strategie zur Terrorismus-Bekämpfung. Mittel- und langfristig werden die zivilen Maßnahmen weitaus wichtiger sein. Für die Koordinierung der Polizei und der Staatsanwaltschaften, für die Austrocknung des finanziellen Hintergrundes der Terrororganisationen, für die Förderung von Toleranz und Rechtsstaatlichkeit wird die EU gebraucht, im Innern und nach außen. Daher muss die EU auch in internationalen Gremien mit einer Stimme sprechen, etwa in Form einer unter den Europäern abgestimmten Politik in den Vereinten Nationen und einer europäisch koordinierten Entwicklungspolitik. Wir brauchen angesichts der Herausforderungen nicht weniger Europa, sondern mehr. 


\section{Die Vereinigung Europas voran bringen}

Die Erweiterung der EU ist die beste Zukunftsinvestition für Deutschland. Sie ist auch ein Stück Wiedervereinigung Europas. Nur sie bietet die Garantie für eine dauerhafte Sicherung von Frieden, Stabilität und Wohlstand. Schon im Beitrittsprozess haben die Kandidaten große Fortschritte bei Rechtsstaatlichkeit und Demokratisierung, bei der Beilegung bilateraler Streitigkeiten, bei der Bewältigung ethnischer Konflikte und bei der Transformation ihrer Volkswirtschaften erreicht und damit zur Stabilisierung Gesamteuropas beigetragen. Sie haben hierfür große Anstrengungen und erhebliche Opfer auf sich genommen. Wenn sich die Beitrittsperspektive nicht rasch konkretisiert, sind Rückschläge in dieser Entwicklung absehbar. Dies dürfen wir im eigenen Interesse nicht zulassen.

Die ökonomischen Chancen der Erweiterung für die bisherigen Mitglieder der EU übersteigen die Risiken bei weitem. Schon jetzt werden durch Handel mit und Investitionen in den Beitrittsländern viele Arbeitsplätze in den westeuropäischen Ländern gesichert. Auch die Angst vor einer Flut von zuwandernden Arbeitnehmern wird weit übertrieben. Alle ernst zu nehmenden Prognosen bestätigen dies. Durch den Beitritt und die Stärkung der Wirtschaftskraft in den Beitrittsländern wird der Auswanderungsdruck eher abnehmen. Andererseits sind wir in manchen Bereichen schon jetzt dringend auf ausländische Arbeitskräfte angewiesen. Daher birgt eine wirkliche geregelte Zuwanderung auch große Chancen.

Die EU und die Beitrittskandidaten müssen sich gemeinsam mit den Bürgern, insbesondere in den betroffenen Grenzregionen in Deutschland und Österreich, auf die Herausforderungen aktiv vorbereiten. Europa darf nicht gegen, sondern muss mit den Bürgern entwickelt werden. Es geht nicht darum, vorhandene Risiken herunterzuspielen und reale Befürchtungen in der Bevölkerung zu missachten. Es geht vielmehr darum, die Diskussion auf eine fundierte Basis zu stellen, unsere Interessen herauszustellen und sich auf die Herausforderungen rechtzeitig vorzubereiten. Die Erweiterungsverhandlungen müssen gleichzeitig mit der Vertiefung der Union zügig weitergeführt werden mit dem Ziel, nach strikter Erfüllung der Beitrittskriterien gut vorbereiteten Kandidaten einen raschen Beitritt so rechtzeitig zu ermöglichen, dass deren Bürger an den Europawahlen 2004 teilnehmen können. Es kommt jetzt vorrangig darauf an, in einem demokratischen Meinungsbildungsprozess eine breite Mehrheit der Bevölkerung für die Ost-Erweiterung zu gewinnen.

\section{Europas Wirtschaft stärken}

Die Europäische Union hat Stabilitäts- und Wohlstandswirkungen nicht nur nach innen, sie muss sich darüber hinaus in einer sich zunehmend globalisierenden Welt behaupten und sich steigender Verantwortung auch für die Weltwirtschaft als ganze stellen.

Mit der Einführung des Euro-Bargeldes ist die europäische Identität für jeden im Wortsinn fühlbar. Durch die einheitliche Währung werden die Vorteile des Binnenmarktes besser ausgeschöpft, werden die Investitionen im Euro-Raum stimuliert und wächst dem Euro-Raum insgesamt ein stärkeres Gewicht in der Weltwirtschaft zu. Der Euro kann aber nur dann ein Erfolg werden, wenn der Binnenmarkt tatsächlich vollendet wird. Dazu gehören vor allem die wirkliche Integration der Geld- und Kapitalmärkte im Euro-Raum sowie eine Abstimmung in der Wirtschafts- und Finanzpolitik, ohne den gesunden Wettbewerb der Volkswirtschaften untereinander zu behindern. Darüber hinaus sind weitere Fortschritte in der Liberalisierung der Märkte, vor allem auch des Arbeitsmarktes, unabdingbar, um die notwendigen Strukturanpassungen in einer sich globalisierenden Welt zu erleichtern.

Auch in Handelsfragen muss die EU künftig mit einer Stimme sprechen. Die Vetomöglichkeit bei Entscheidungen des Rates im Rahmen der Handelspolitik muss so schnell wie möglich beseitigt werden, damit die einzelnen EU-Mitgliedstaaten bei den WTO-Verhandlungen nicht gegeneinander ausgespielt werden können.

In der Agrarpolitik müssen die Landwirte von Bürokratie entlastet und wieder zu Unternehmern gemacht werden. Wir brauchen keine Planwirtschaft, sondern wieder eine marktorientierte Landwirtschaft. Unternehmerische Landwirte sollen in Zukunft einen wesentlichen Teil ihres Einkommens am Markt erzielen können sowie Zusatzeinkommen für erbrachte Landschaftspflege und Sonderleistungen erhalten. Die Möglichkeiten der Kofinanzierung müssen dabei im Sinne des Subsidiaritätsprinzips und im Sinne einer faireren Lastenverteilung ausgeweitet werden.

Auch die Strukturpolitik muss reformiert werden, nicht nur aus finanziellen Gesichtspunkten. Die neuen Prioritäten müssen bei der Verbesserung von Infrastruktur und Bildung sowie der Förderung von unternehmerischer Eigeninitiative und der Entwicklung von wettbewerbsfähigen Klein- und Mittelbetrieben liegen, um Europa wieder innovativ und wettbewerbsfähig mit anderen Weltregionen werden zu lassen.

\section{Eine Verfassung für ein Europa der Bürger}

Die europäische Integration ist an einem Punkt angelangt, an dem es immer klarer wird, dass ihre Fortführung nur mit einer deutlichen Zieldefinition oder zumindest einer klaren Richtungsweisung akzeptiert wird. Es ist an der Zeit, das europäische Gesellschaftsmodell, beruhend auf den Grundprinzipien Freiheit, Demokratie, Achtung der Menschenrechte und Grundfreiheiten, Rechtsstaatlichkeit, Gewaltenteilung, Subsidiarität und Solidarität, als föderal verfasste Europäische Union vertraglich $\mathrm{zu}$ verankern. $\mathrm{Zu}$ klären sind dabei das Verhältnis der Nationalstaaten zu den europäische Institutionen auch im Sinne einer klaren, sinnvollen Kompetenzabgrenzung zwischen europäischer, nationaler und regionaler Ebene, das Verhältnis der europäischen Organe untereinander, die Frage der demokratischen Legitimität und Kontrolle von Entscheidungen auf europäischer Ebene sowie die dafür notwendige Transparenz der Entscheidungsfindung. Bei der hierfür erforderlichen Neuformulierung und ggf. Zweiteilung der Europäischen Verträge muss darauf geachtet werden, dass sie zumindest in einem Allgemeinen Teil auch für interessierte Laien verständlich sind. Allein verständliche, nachvollziehbare und demokratisch kontrollierte Prozesse in der Europäischen Union können der sich verstärkenden Europaskepsis entgegenwirken. 
Nun gilt es, konkret eine europäische Verfassung oder einen europäischen Verfassungsvertrag zu erarbeiten. Die Europäische Grundrechte-Charta wird dessen Grundstein sein können. Ihr kommt Modellcharakter auch in ihrer Entstehungsweise zu: Das Konventmodell unter weitestgehender Beteiligung der nationalen Parlamente und des europäischen Parlaments und unter Einbeziehung der Öffentlichkeit sollte zur Erarbeitung des Verfassungsvertrages genutzt werden. Der Konvent soll sich eigene Regeln für Struktur und Arbeitsweise geben und einen möglichst weit ausgearbeiteten Verfassungstext vorlegen, der Optionen nur da enthält, wo es unabdingbar ist. Die Regierungskonferenz 2004 soll in möglichst unmittelbarem Anschluss daran auf Grundlage des Textes, den der Konvent vorschlägt, den Verfassungsvertrag fertig stellen. Am Ende muss die Verfassung von den Bürgern der Europäischen Union durch ein Referendum bestätigt werden. Mit diesem Verfahren - Konvent und Referendum wird eine neue Stufe der europäischen Integration erreicht.

\section{5. Äußere Sicherheit schafft Sicherheit auch im In- nern}

Seiner Verantwortung für Krisenprävention und Friedenssicherung kann Deutschland nur durch eine Gemeinsame Außen- und Sicherheitspolitik der Europäischen Union gerecht werden, die diesen Namen verdient. Wir brauchen den entschlossenen politischen Willen aller Mitgliedstaaten, mit einer Stimme zu sprechen und gemeinsam zu handeln, wenn Frieden und Stabilität in Europa bedroht sind.

\section{Die Europäische Sicherheitspolitik ausbauen}

Die europäische Sicherheits- und Verteidigungspolitik (ESVP) muss konsequent ausgebaut werden. Ein Konkurrenzverhältnis zur Nordatlantischen Allianz, die auch künftig Motor und Garant einer breit angelegten europäischen Sicherheitsarchitektur bleiben muss, darf nicht entstehen. Vielmehr ist eine strategische Partnerschaft zwischen EU und NATO bei der Krisenbewältigung das Ziel, in der die EU nur dann tätig wird, wenn sich die NATO als ganze nicht engagieren kann oder will. Eine funktionierende ESVP stärkt nicht nur Europa, sondern auch die Allianz. Ein Europa mit einer funktionierenden ESVP ist auch ein besserer Partner für die USA. Die ESVP hat mit den Einsätzen im ehemaligen Jugoslawien ihre ersten Bewährungsproben bestanden, obwohl ihre Instrumente noch nicht ausgereift sind. Daher sollte schon jetzt darüber nachgedacht werden, wie die europäischen Einsatzkräfte über die ihnen bisher zugewiesenen sog. »Petersberg-Aufgaben« (humanitäre Aufgaben und Rettungseinsätze, friedenserhaltende Aufgaben sowie Kampfeinsätze bei der Krisenbewältigung einschließlich friedensschaffender Maßnahmen) hinaus zu einer echten europäischen Spezialtruppe weiterentwickelt werden können.

Die Vereinigten Staaten von Amerika bleiben unser wichtigster Partner außerhalb Europas. Im Rahmen dieser Partnerschaft muss es möglich sein, Interessengegensätze offen und fair $\mathrm{zu}$ behandeln und auszugleichen.

\section{Die NATO stärken}

Die NATO-Verbündeten haben unmittelbar nach den brutalen Terrorakten auf das World Trade Center und das Pentagon den USA eindrucksvoll ihre Solidarität versichert. Die Atlantische Allianz hat wenig später einmütig und zum ersten Mal den Bündnisfall festgestellt. Die terroristischen Angriffe auf New York und Washington wurden als Angriff auf die freie Welt gewertet. Deshalb ist die NATO gefordert. Politische und militärische Planung, Organisation, Koordination und Führung des Kampfes gegen den Terrorismus unter Ausschluss der NATO wäre der falsche Weg. Die Qualitäten des Bündnisses, während des Kalten Krieges eindrucksvoll unter Beweis gestellt, dürfen nicht ungenutzt bleiben. Deutschland, das im Kalten Krieg die Hauptlast der westlichen Verteidigung neben den USA trug, wird auch in Zukunft seiner Verantwortung im Rahmen des Bündnisses gerecht werden müssen.

Die Frage des höchstmöglichen Nutzens und des geringsten Schadens für die gesamteuropäische Stabilität muss die Richtschnur der Erweiterungsentscheidung sein. Oberstes Ziel muss die uneingeschränkte Aufrechterhaltung der Handlungsfähigkeit des Bündnisses als Garant euro-atlantischer Sicherheit bleiben. Ein Bündnis, das durch die Erweiterung in seiner Leistungsfähigkeit beeinträchtigt wird, nützt weder den Neumitgliedern noch den zu stabilisierenden Regionen. Daher sollte die sicherheitspolitische Kompatibilität der Kandidaten zu einem Beitrittskriterium werden.

\section{Internationale Ordnungspolitik für Vertrauen und Sicherheit}

Jenseits der europäisch-atlantischen Stabilitätszone gibt es ein wachsendes terroristisches Gewaltpotential. Eine zukunftsweisende außenpolitische Strategie muss daher die Stärkung der internationalen Zusammenarbeit, vor allem im Rahmen der Vereinten Nationen und ihrer regionalen Abmachungen, in den Vordergrund stellen. Globale und regionale Ordnungspolitik sowie Konfliktprävention und Regulierung lassen sich nicht im nationalen Alleingang und erst recht nicht kurzfristig erreichen, sondern erfordern eine langfristige Strategie. Außenpolitik darf nicht erst auf Fernsehbilder reagieren, sondern muss durch stetes präventives Eintreten für eine internationale Konfliktvermeidung ersetzt werden. Deutschland muss hier eine aktive und maßgebliche Rolle übernehmen. Dabei muss sich Deutschland vor Sonderwegen hüten. Unsere Verbündeten und Nachbarn erwarten das, was wir leisten können und nach unseren Fähigkeiten auch leisten sollten.

\section{Die Vereinten Nationen umfassend stärken}

Mit seiner eindeutigen und schnellen Reaktion nach dem 11. September hat der Sicherheitsrat der Vereinten Nationen (VN) gezeigt, dass er als zentrales weltinnenpolitisches Instrument alternativlos ist. Als einzige weltumspannende und handlungsfähige Organisation bieten die Vereinten Nationen einen geeigneten Rahmen für die Umsetzung präventiver Strategien. Die politische Zuwendung der USA zur Weltorganisation, die Zahlung der rückständigen Beiträge, die 
Verleihung des Friedensnobelpreises an Kofi Annan und die seit Mazedonien gewährleistete politische Funktionsfähigkeit des Sicherheitsrates bieten zusammen mit dem neuen weltpolitischen Aufbruch gegen den Terrorismus eine günstige Ausgangslage für die weitere Stärkung der Vereinten Nationen. Als drittgrößter Beitragszahler steht Deutschland hier in besonderer Verantwortung. Das VN-System muss in allen seinen Bereichen ausgebaut und nachhaltig gestärkt werden. Dies bedeutet nicht nur erhebliche zusätzliche finanzielle Leistungen, es bedeutet auch die Bereitschaft zur Übertragung nationaler Kompetenzen auf die multilaterale Ebene. Die Schaffung des Weltstrafgerichtshofes ist hier ein erster wichtiger Schritt. Weitere müssen folgen.

Der Afghanistan-Einsatz, wie schon vor ihm die Einsätze auf dem Balkan, zeigen, dass es sich bei der zukünftigen weltweiten Streitschlichtung nicht nur um Aktionen einzelner Staaten handeln kann, sondern dass die Staatengemeinschaft als Ganzes gefordert ist. Wenngleich den regionalen Sicherheitsbündnissen, insbesondere der NATO, zukünftig eine zentrale Koordinierungsfunktion bei derartigen Einsätzen zukommt, müssen die Vereinten Nationen in die Lage versetzt werden, unmittelbar und aus eigenen Kräften streitschlichtend oder friedensschaffend eingreifen zu können. Die in dem sogenannten »Brahimi-Bericht« vorgeschlagenen Reformen müssen dringend umgesetzt werden. Gerade jetzt kommt es darauf an, den internationalen Terroristen die Stirn zu bieten und ihnen zu zeigen, dass sie in der Gemeinschaft zivilisierter Staaten keinen Platz haben. Der VN-Sicherheitsrat hat dies bereits klargestellt. Die Generalversammlung sollte ihm zügig folgen und die Voraussetzungen für die Verabschiedung einer umfassenden UNO-Konvention gegen den Terrorismus schaffen.

Die deutsche VN-Politik darf sich daher nicht auf den eher halbherzig vorgetragenen Wunsch einer Mitgliedschaft im Sicherheitsrat beschränken. Wer mitreden will, muss auch mithandeln. Dies setzt sowohl größeres Engagement an den VN-Reformbestrebungen, eine aktivere deutsche VN-Personalpolitik, aber auch eine aktivere Beteiligung Deutschlands an VN-Blauhelmmissionen voraus. Deutschland muss daher gemeinsam mit unseren Partnern in der Europäischen Union eine Initiative zur Stärkung der Vereinten Nationen als zentralem Instrument zur Bewältigung der neuen globalen Herausforderungen ergreifen.

Regionale Konflikte sollten in den Regionen gelöst werden. Die Vereinten Nationen müssen für diesen Zweck handlungsfähiger werden. Um zukünftige Blockierungen des Sicherheitsrates bei dringendem Handlungsbedarf der Staatengemeinschaft zur Lösung regionaler Krisen zu vermeiden, müssen die regionalen Abmachungen mit dem Ziel gestärkt werden, mehr Verantwortung für friedenserhaltende Maßnahmen zu übernehmen.

\section{Der OSZE mehr Gewicht beimessen}

Die KSZE war das Erfolgsmodell für die Überwindung der Spaltung der Welt in Ost und West durch den schrittweisen Aufbau von gegenseitigem Vertrauen und Möglichkeiten zur Zusammenarbeit. Die aus der KSZE hervorgegangene OSZE ist die einzige Sicherheitsinstitution, die alle europäischen
Staaten inklusive Russland, die USA, Kanada sowie die neuen Staaten des Kaukasus und Zentralasiens umfasst. Dennoch wird der OSZE heute nicht das Gewicht eingeräumt, das nötig wäre, um Konflikte präventiv zu vermeiden. Die OSZE muss eine neue ernsthaftere Rolle in der deutschen Außenpolitik spielen.

Das bewährte KSZE/OSZE-Modell (vertrauensbildende Maßnahmen, Stärkung rechtsstaatlicher Strukturen, regionale $\mathrm{Zu}$ sammenarbeit, Abrüstung und Rüstungskontrolle, Konfliktverhütung, Minderheitenschutz und Flüchtlingsrückkehr, Krisenbewältigung und Frühwarnung) sollte Grundlage einer dauerhaften politischen Lösung regionaler Konflikte werden.

\section{Den Balkan politisch stabilisieren}

Dank großer, auch militärischer, Anstrengungen konnte ein derzeit halbwegs friedlicher Zustand auf dem Balkan erreicht werden. Nun müssen aber auch politische Lösungen mit Hochdruck angestrebt werden. Militärische Präsenz plus Geberkonferenzen reichen bei weitem nicht aus. Es bietet sich an, dass der Balkan vom Kalten Krieg lernt und das Erfolgskonzept KSZE zur Anwendung kommt. Notwendig ist eine erkennbare Gesamtstrategie für Südosteuropa, die in eine umfassende Südosteuropa-Konferenz münden muss. Eine solche Konferenz muss unter dem Dach der OSZE einberufen werden und dieselben Ziele verfolgen, die seinerzeit die KSZE anstrebte. Die betroffenen Staaten und Regionen sollten sich in einer solchen Konferenz für Sicherheit und Zusammenarbeit in Südosteuropa (KSZSE) darauf verpflichten, alle offenen Fragen wie Minderheitenstatus, Vertriebenenrückkehr, Aufbau rechtsstaatlicher Strukturen und Rüstungskontrolle auf den Tisch zu bringen und im Sinne eines kontinuierlichen, langfristigen Abstimmungsprozesses zu lösen. Die bereits im Rahmen des KSZE-Prozesses erfolgreichen Instrumente wie vertrauensbildende Maßnahmen, Streitschlichtung, regionale Kooperation sollten hierbei länderspezifisch geklärt und eingesetzt werden.

\section{Den Nahost-Friedensprozess voranbringen}

Deutschland und Europa müssen eine aktivere Rolle im Nahost-Friedensprozess einnehmen. Dabei geht es nicht in erster Linie um Vermittlerrollen, sondern um ein stärkeres europäisches Engagement für Lösungsoptionen an der Seite der USA. Die Terroranschläge gegen Israel sind vollkommen inakzeptabel. Der Terror muss gestoppt werden. Das Existenzrecht Israels ist unantastbar. Auf der anderen Seite muss jedoch auch Israel einsehen, dass die Fortsetzung der bisherigen Siedlungspolitik einer Friedenslösung im Wege steht und ein Palästinenserstaat die beste Sicherheitsgarantie gerade für Israel bedeuten kann. Die internationale Staatengemeinschaft, die UNO, die USA, die EU und auch Russland müssen alles in ihrer Kraft stehende tun, damit der in Oslo so hoffnungsvoll begonnene Verhandlungsprozess wieder aufgenommen werden kann.

Darauf aufbauen sollte eine Konferenz für Sicherheit und Zusammenarbeit im Nahen Osten (KSZNO) unter Schirmherrschaft der Vereinten Nationen und der Europäischen 
Union bei Beteiligung aller betroffenen Länder der Region, an deren Ende eine belastbare Friedensregelung mit vertrauensbildenden Maßnahmen, Abrüstung und Rüstungskontrollverträge, Konfliktverhütung, Krisenbewältigung, Frühwarnung etc. stehen muss. Grundlage für Frieden in der Region ist sowohl das unantastbare Existenzrechts Israels als auch die Schaffung eines unabhängigen Palästinenserstaates.

\section{Die euro-atlantischen Beziehungen vertiefen}

Die freundschaftliche Partnerschaft Deutschlands mit den Vereinigten Staaten von Amerika ist weit mehr als politische Vernunft, sie zeichnet sich durch eine tiefgehende Wertegemeinschaft und gewachsenes gegenseitiges Vertrauen aus. Als größtem Land der Europäischen Union und wichtigstem NATO-Partner der USA kommt Deutschland eine besondere Verantwortung zu. Angesichts zunehmender sicherheits- und handelspolitischer Herausforderungen stehen die transatlantischen Beziehungen vor einer wichtigen Bewährungsprobe. Bananen, Hormonrindfleisch, gentechnisch modifizierter Mais, Soja und Raps, die Helms-Burtonund die D'Amato-Gesetze, Boeing und Airbus und neuerdings Stahlimporte sowie das amerikanische Raketenabwehrsystem »National Missile Defense « (NMD) und die Perspektive einer eigenständigen europäischen Sicherheits- und Verteidigungspolitik (ESVP) sind Stichworte, die ein wachsendes euro-atlantisches Irritationspotenzial illustrieren.

Europa und Amerika haben jedoch mehr gemeinsame Interessen als jeder allein mit irgendeinem Partner in der Welt. Gerade im Zeichen der Globalisierung nicht nur von Handel und Information, sondern auch von Sicherheitsrisiken, müssen die euro-atlantischen Partner näher zusammenrücken. Eine enge und vertrauensvolle Zusammenarbeit zwischen diesen beiden mit Abstand wichtigsten Akteuren der Weltpolitik ist unerlässlich. Bislang ist es jedoch nicht gelungen, einen Rahmen zu finden, der den wachsenden euro-atlantischen Herausforderungen standhält. Die hohen Erwartungen an die »Neue Transatlantische Agenda " haben sich nicht erfüllt. Die halbjährlichen Gipfeltreffen, politische Konsultationen und auch der »Business-Dialog « europäischer und amerikanischer Unternehmen sind zwar nützliche Ansätze, ergeben jedoch noch kein tragfähiges Netzwerk.

Ein mit Frankreich abgestimmtes Konzept zur Vertiefung der euroatlantischen Beziehungen ist vonnöten. Dabei geht es ebenso um Handlungsoptionen für die zügige Verwirklichung eines transatlantischen Marktes einschließlich WTO-konformer Streitschlichtungsmechanismen als auch um eine neue Qualität der Sicherheitspartnerschaft zwischen Amerika und Europa.

Zusätzlich zu den bisherigen Pfeilern unserer Transatlantischen Sicherheits- und Wertepartnerschaft sollte nun mit Hochdruck an einer gemeinsamen Freihandelszone gearbeitet werden. Nordamerika ist bereits einige Schritte vorangekommen mit dem Aufbau einer Amerikanischen Freihandelszone, die bisher erreichten Freihandelsabkommen zwischen Europa und Nord- sowie Südamerika dürfen dabei jedoch nicht in Frage gestellt werden. Wichtig wären nun parallele Bestrebungen für WTO-konforme gemeinsame Handelserleichterungen. Wir brauchen eine transatlantische Freihandelszone als gemeinsamen Wachstumsmotor zwischen Europa sowie Nord- und Südamerika.

\section{Den Dialog mit Russland intensivieren}

Durch seine engagierte Mitwirkung an der globalen Koalition gegen Terrorismus hat Russland seine Bereitschaft unter Beweis gestellt, mehr weltpolitische Verantwortung tragen $\mathrm{zu}$ wollen. Dies ist eine große Chance für die Gestaltung einer gesamteuropäischen Friedensordnung, bei der Russland eine zentrale Rolle zufällt. Die Bundesregierung muss nun konstruktive, realistische Vorschläge unterbreiten, wie Russland am zweckmäßigsten in die euro-atlantischen Sicherheitsstrukturen eingebunden werden kann. Bundeskanzler Schröder hat Deutschland als Motor im Verhältnis zwischen EU und Russland bezeichnet. Der intensivierte politische Dialog scheint auf der Ebene der Regierungschefs und der wichtigsten Fachministerien zu funktionieren. Dennoch erfüllt er die Erwartungen nicht, weil entweder die Interessen divergieren oder interne Entscheidungsprozesse noch nicht abgeschlossen sind. Wer Motor sein will, muss aber auch die Richtung angeben können, in die man sich zu bewegen gedenkt.

In der wichtigen Frage der Rückführung der sogenannten »Beutekunst« gibt es keine Bewegung. Die Gründung einer »Deutsch-Russischen Kulturstiftung«, der die Kulturgüter, soweit sie im Eigentum der öffentlichen Hand stehen, übertragen werden, könnte hier zu einer Lösung im beiderseitigen Einvernehmen führen.

Eine gute partnerschaftliche Beziehung muss auch kritischen Diskussionen standhalten. Die Intensivierung der Beziehung Deutschlands zu Russland muss auch durch die Erörterung schwieriger Themen wie z. B. der Menschenrechtssituation in Tschetschenien begleitet werden. Russland braucht eine europäische Perspektive, sein wirtschaftlicher Erfolg liegt in unserem Interesse und sein Mitwirken in Sicherheitskonzepten ebenso.

\section{Eine europäische Afrika-Strategie entwickeln}

Seit dem Ende der Ost-West-Konfrontation haben sich neue Ansätze für einen nachhaltigen Strukturwandel durch Reformen und für einen intensiven politischen Dialog mit den afrikanischen Staaten ergeben. Die Situation ist sehr differenziert, einige Staaten zeigen große wirtschaftliche wie gesellschaftliche Fortschritte, während andere Staaten schlechter dastehen als vor 40 Jahren. Entwicklungspolitik muss hieraus die Konsequenzen ziehen und insbesondere die viel versprechenden neuen afrikanischen Initiativen zur Übernahme von mehr Eigenverantwortung, etwa im Rahmen der Neuen Partnerschaft für Afrikanische Entwicklung (NEPAD), übernehmen. In diesem Bereich hätte die Bundesregierung eine wirkliche konzeptionelle Aufgabe.

Es ist höchste Zeit, die Vorgaben des Amsterdamer Vertrages umzusetzen und eine gemeinsame europäische AfrikaStrategie im Rahmen der Gemeinsamen Außen- und Sicherheitspolitik zu entwickeln. Dies betrifft auch eine kohären- 
te gemeinsame europäische Entwicklungspolitik, in deren Mittelpunkt Konzepte zur nachhaltigen Armutsbekämpfung durch Demokratieberatung, Ausbildung und Informationstechnologie sowie verstärkte Maßnahmen zur Bekämpfung und Prävention von AIDS stehen müssen. In diesen Kontinent darf nicht weiter so hinein gestolpert werden, wie das in den letzten Jahren der Fall war.

\section{Die Beziehungen zu Lateinamerika dynamisieren}

Wie mit keiner anderen Region der Welt ist Europa mit Lateinamerika durch eine Vielzahl von historischen, kulturellen, religiösen und ethnischen Beziehungen verbunden. Dennoch haben die Beziehungen bei weitem nicht den notwendigen Stellenwert. Gleichzeitig ist eine stärkere Hinwendung Lateinamerikas an Nordamerika festzustellen. Am deutlichsten zeigt sich dies an dem proportionalen Rückgang von Im- und Exporten der EU mit Lateinamerika.

Die Beziehungen Deutschlands und der Europäischen Union mit Lateinamerika bedürfen dringend eines neuen Elans. Das gilt besonders für die Beziehungen zwischen der EU und MERCOSUR. Als maßgeblich von der Außenverflechtung abhängige Volkswirtschaft hat Deutschland hier eine besondere Verantwortung gegenüber den EU-Partnern, um auf den dringend erforderlichen Abbau von tarifären und nichttarifären Handelshemmnissen im Warenaustausch mit Lateinamerika hinzuwirken. Eine weitere Enttäuschung der großen Erwartungen der Lateinamerikaner an einen intensiven Austausch mit der Europäischen Union würde zwangsläufig zu einer Verstärkung der Zusammenarbeit mit NAFTA zu Lasten Europas führen. Die Verbesserung von bilateralen Handelsbeziehungen darf nicht zu Lasten Dritter gehen. Mit Hilfe eines WTO-konformen transatlantischen Dreiecks zwischen MERCOSUR, EU und NAFTA könnte den Interessen dieser drei Weltregionen gedient werden, ohne bereits erreichte Handelserleichterungen zu gefährden.

\section{Neue Perspektiven für die Asienpolitik entwickeln}

Die deutsche Asienpolitik muss der wachsenden wirtschaftlichen, politischen und demografischen Bedeutung dieses Kontinents gerecht werden. 26 Prozent des Weltsozialproduktes werden in Asien erwirtschaftet, 56 Prozent der Weltbevölkerung, aber auch 66 Prozent aller Armen leben in der Region. Das Interesse Europas an Asien kann und darf daher nicht nur ökonomischer Natur sein. Die bislang von Afghanistan ausgehende Destabilisierung, die potenziellen Krisenregionen östlich des Kaspischen Meeres, der Kaschmir-Konflikt, die schwierigen Entwicklungen auf der koreanischen Halbinsel, Auflösungserscheinungen des indonesischen Inselreiches, die Problembereiche eines an Bedeutung gewinnenden China mit seinen Nachbarn, das sich zunehmend modernisierende Indien, die erkennbaren Bestrebungen Pakistans zu einer neuen internationalen Rolle und die in diesem Feld auch aufgeworfenen nuklearstrategischen Grundsatzfragen, all dies verlangt nach einem überarbeiteten konzeptionellen Ansatz, der den neuen asiatischen Herausforderungen gerecht wird. Einem Großteil der asiatischen Länder, allen voran China und Indien, wird die bisherige Entwicklungspolitik nicht gerecht. In diesen Ländern gibt es zwar Regionen, die dringend einer Entwicklung bedürfen, gleichzeitig jedoch auch Wachstumszentren mit erheblicher Impulswirkung, die weit über die Region hinaus reicht.

Auch das Verhältnis zwischen der EU und Asien bedarf dringend neuer Anstöße für den systematischen Ausbau der Beziehungen zwischen beiden Regionen: Verstärkter Austausch zur Verringerung der kulturellen und gesellschaftlichen Distanz, politischer Dialog einschließlich sicherheitspolitischer Fragen (Nichtverbreitung und Abrüstung), weiterer Ausbau des gegenseitigen Handels und der Investitionen, Vertiefung des Menschenrechtsdialogs, Umweltschutz, organisiertes Verbrechen und Menschenrechte, institutionelle Verknüpfung zwischen ASEAN und EU, Verstärkung des ASEM-Prozesses (bisher nur ASEF und Umwelttechnologiezentrum Bangkok), Ausbau von Parlamentariernetzwerken, Studentenaustausch und Austausch von Jungunternehmern.

\section{Die Außenwirtschaft besser fördern}

Außenpolitik ist Interessenpolitik in einem umfassenden Sinn. Gute Außenbeziehungen erleichtern Handel und Investitionen und damit die Schaffung von Arbeitsplätzen auf beiden Seiten. Die Außenwirtschaftsförderung muss wieder zu einem der zentralen Punkte der deutschen Außenpolitik werden. Vor allem der deutsche Mittelstand, der über kein eigenes Netz von Auslandsvertretungen verfügt, ist auf flankierende Unterstützung durch die deutsche Außenhandelspolitik angewiesen. Eine gelegentliche Beteiligung von Wirtschaftsvertretungen bei Ministerreisen reicht nicht aus. Gefragt ist ein aktives Engagement jedes einzelnen Mitarbeiters sowie die bessere Vernetzung und Koordinierung zwischen dem auswärtigen Dienst und den Außenvertretungen der deutschen Wirtschaft. Gerade im Zeitalter der Globalisierung hängt die Wettbewerbsfähigkeit des Wirtschaftsstandortes Deutschland maßgeblich von dem Erfolg deutscher Unternehmen auf den Weltmärkten ab. Damit die deutschen Auslandsvertretungen ihre Rolle als effiziente Dienstleister für die Unterstützung der deutschen Wirtschaft wahrnehmen können, müssen auch endlich die kleinkarierten Ressorteifersüchteleien innerhalb der Bundesregierung beigelegt werden. Deutschland muss über das Auswärtige Amt als klassischem Querschnittsressort nach außen mit einer Stimme in allen Fachbereichen, also auch in der Außenwirtschaftspolitik, sprechen.

Gerade angesichts der terroristischen Bedrohungen muss die Staatengemeinschaft insbesondere auch ihren gemeinsamen Willen zur Festigung und Ausweitung des Freihandels deutlich machen. Das Desaster von Seattle hat gezeigt, dass es zu einer multilateral abgestimmten Handelspolitik keine Alternative gibt. Mehr Handel und verbesserter Marktzugang für Entwicklungsländer ist die wirkungsvollste Entwicklungshilfe. Dabei kommt den euroatlantischen Beziehungen eine zentrale Rolle zu. Europa und Nordamerika können die großen weltweiten Herausforderungen nur meistern, wenn sie auch in handelspolitischen Fragen näher zusammenrücken.

Deutschland darf hier nicht bremsen, sondern muss zum Meinungsführer innerhalb der EU werden und sich aktiv für den Abbau der noch verbliebenen Handelshemmnisse, 
auch in sensiblen Sektoren wie Agrar und Textil, einsetzen. Das Hauptaugenmerk sollte hier bei Maßnahmen zur Schlichtung transatlantischer Handelsstreitigkeiten, Liberalisierung audiovisueller Dienstleistungen, WTO-Konformität von Exportsubventionen, Abbau nichttarifärer Handelshemmnisse, Verbesserung des Marktzuganges für landwirtschaftliche Produkte aus Entwicklungsländern, Kohärenz zwischen europäischer Entwicklungs-, Agrar- und Handelspolitik liegen. Die EU-Kommission muss endlich ein Mandat zur WTO-Verhandlungsführung auf der Grundlage von Mehrheitsbeschlüssen bekommen. Die Europäische Zentralbank sollte ermächtigt werden, ihre Politik einer engeren $\mathrm{Zu}$ sammenarbeit und Abstimmung mit der Federal Reserve Bank mit dem Ziel einer Stabilisierung der internationalen Finanzmärkte zu verstärken.

\section{Innovative Lösungen für die Auswärtige Kulturpoli- tik}

Gerade in Zeiten rückläufiger Haushaltsmittel muss auch die Auswärtige Kulturpolitik kreative und innovative Lösungen finden, um ihrem Auftrag gerecht zu werden. Dies bedeutet zum einen den zielgenaueren und effizienteren Einsatz der wenigen vorhandenen Mittel, zum anderen sollten in der Auslandskulturarbeit auch stärker als bisher nichtöffentliche Träger komplementär hinzugezogen werden. Im Rahmen der Reform der Auswärtigen Kulturpolitik (AKP) muss ein programmatischer Schwerpunkt auf den transkulturellen Dialog, insbesondere zwischen den Regionen, Religionen und Kulturen gelegt werden. Die AKP muss durch Verbreitung neuer pluralistischer Bildungsinhalte, Kulturdialog und Einbindung der Zivilgesellschaft in offene Netzwerke der internationalen Zusammenarbeit einen Beitrag zum Abbau von Feindbildern und Vorurteilen und damit auch zur Bekämpfung des Terrorismus leisten.

In diesem Rahmen ist es unabdingbar, das Auswärtige Amt, die politischen Stiftungen, die Mittler der Kulturpolitik (Goethe-Institute, DAAD, Kulturstiftung etc.) und andere einschlägig tätige Nichtregierungsorganisationen finanziell nicht weiter zu beschneiden, sondern im Gegenteil wieder stärker zu fördern. Die Vermittlung einer kulturellen deutschen Identität im Ausland und die Zusammenführung der deutschen Kultur mit dem kulturellen Umfeld des jeweiligen Gastlandes liegt auch im Interesse der sich weltweit engagierenden deutschen Unternehmen, wie sich etwa bei den Schwierigkeiten der Gewinnung ausländischer Spezialisten aufgrund von Sprachschwierigkeiten zeigt. Eine »Public Private Partnership« ist daher in der Auswärtigen Kulturpolitik ebenso legitim, wie sie in der entwicklungspolitischen Zusammenarbeit erforderlich ist. Im Rahmen der Verschlankung der Trägerstrukturen der Auswärtigen Kulturpolitik muss ferner verstärkt dazu übergegangen werden, die Programmarbeit im Rahmen lokaler Strukturen abzuwickeln. Dies gilt für den Sprachunterricht der Goethe-Institute ebenso wie für die deutschen Auslandsschulen, die verstärkt auf das in vielen Ländern vorhandene erhebliche Potential deutscher Ortskräfte zurückgreifen sollten.

Auch innerhalb Deutschlands kann ein Beitrag zur Auswärtigen Kulturpolitik geleistet werden. Hier geht es insbesondere um die Verbesserung der Rahmenbedingungen für ausländische Studenten: Werbung für den Studienstandort Deutschland, von der deutschen Wirtschaft kofinanzierte Stipendienangebote, Vereinfachung der Stipendienauswahlverfahren, Aufbau fremdsprachiger Studiengänge an deutschen Hochschulen, Verbesserung studienbegleitender Betreuung für ausländische Studierende, Aufbau von OnlineStudiengängen.

\section{Außenpolitische Instrumente stärken}

Deutschland kann es sich nicht leisten, aus kurzfristigen haushaltspolitischen Erwägungen seinen Auswärtigen Dienst »kaputt zu sparen«. Im Gegenteil: Gerade jetzt muss der Auswärtige Dienst in allen seinen Teilen finanziell und personell in die Lage versetzt werden, den neuen weltweiten Herausforderungen wirkungsvoll $\mathrm{zu}$ begegnen. Die Außenrepräsentanz der Bundesrepublik Deutschland - dazu gehören neben dem Auswärtigen Dienst die Akteure in der Entwicklungszusammenarbeit, die politischen Stiftungen, die Kulturmittler, das Netzwerk der Wirtschaftsförderung, die im Ausland tätigen Nichtregierungsorganisationen - muss drastisch gestärkt werden. Dafür ist Geld notwendig, aber auch der Einsatz von Fantasie und Kreativität bei der Vernetzung, der Koordinierung und dem personellen Austausch.

Eine ersatzlose Schließung von Auslandsvertretungen und Goethe-Instituten schadet deutschen Interessen und darf nicht weiter hingenommen werden. Vielmehr sollten endlich auch in der Gemeinsamen Europäischen Außen- und Sicherheitspolitik die Weichen für gemeinsame Strukturen in sogenannten Drittländern geschaffen werden.

Eine effiziente und glaubwürdige Wahrnehmung deutscher Interessen im Ausland setzt nicht nur einen gut ausgestatteten Auswärtigen Dienst, sondern auch umfassende Querschnittskompetenzen des Auswärtigen Amtes voraus. Es ist daher dringend erforderlich, dass das Auswärtige Amt seine traditionelle Koordinierungsrolle zurückerhält und der zunehmenden Übernahme außenpolitischer Kompetenzen durch andere Ressorts Einhalt geboten wird. In Anbetracht der Tatsache, dass unsere außenpolitischen Beziehungen zu zwei Dritteln der Länder im wesentlichen entwicklungspolitische Beziehungen sind und dass Entwicklungspolitik ein wichtiger Teil der Außenpolitik ist, ist es überdies geboten, die Tätigkeitsfelder von BMZ und AA durch Zusammenlegung der Ministerien zusammenzuführen.

\section{Menschenrechte ernst nehmen}

Tschetschenien, China, Todesstrafe und Tibet: Selbst dort, wo Rot-Grün neue Schwerpunkte setzen wollte, bei den Menschenrechten, ist bislang außer wortgewaltiger Rhetorik keine nachhaltige Initiative erkennbar. Hier wird Ambivalenz und Inkohärenz grüner Politik ebenso deutlich wie beim Export der Hanauer Plutonium-Fabrik nach Russland oder bei den Hermes-Bürgschaften für den Drei-Schluchten-Staudamm in China. Dies gilt auch für den kalkulierten Dissens über Panzerlieferungen im Bundessicherheitsrat. Der wirksame Schutz von Menschen- und Minderheitsrechten und der Dialog der Kulturen müssen überdies im Zentrum einer präventiven Strategie gegen Terrorismus stehen. Die Bundesregierung 
muss daher den globalen Menschenrechtsdialog mit dem Ziel verstärken, die mentale Kluft zwischen aufgeklärter Moderne und fundamentalistischem Traditionalismus zu überbrücken und die Kultur der Intoleranz durch die Kultur der Toleranz zu ersetzen, damit sich die inneren Konflikte in diesen Gesellschaften nicht gewaltsam nach außen entladen.

Schwerpunkte deutscher Menschenrechtspolitik müssen neben der deutlichen Kritik an Menschenrechtsdefiziten u. a. in Tschetschenien und China der Einsatz gegen die Folter von Kindern, das Engagement für die Abschaffung der Todesstrafe, der Schutz der weltweit zunehmenden Zahl von Binnenvertriebenen, das Eintreten für die Einbeziehung von transnationalen Unternehmen in die Verantwortung für die Menschenrechte sowie die weltweite Verbesserung von Haftbedingungen sein.

Die Schaffung des Ständigen Internationalen Strafgerichtshofes für Kriegsverbrechen, Völkermord und Verbrechen gegen die Menschlichkeit ist ein wichtiger Baustein zum Aufbau einer weltweit verbindlichen Judikative zur Durchsetzung von Völkerrechtsnormen.

Außenpolitik und Wirtschaft müssen gemeinsam Wege zur Verstärkung der normativen, positiven und punitiven Ansätze zur Durchsetzung von Menschenrechten suchen. Das vielversprechende Modell einer OECD-Konvention gegen Korruption muss nun auch in die Tat umgesetzt werden.

Wolfgang Gehrcke

\section{»Linke« Außenpolitik: Frieden und Gerechtigkeit}

Wolfgang Gehrcke, MdB, Stell-
vertretender Vorsitzender und
außenpolitischer Sprecher der
PDS-Bundestagsfraktion, Ber-
lin

Michael Gorbatschow hatte als Generalsekretär der einst mächtigen Kommunistischen Partei der Sowjetunion das Ende des Kalten Krieges mit der Einsicht eingeläutet, dass die globalen Probleme der Menschheit globale Kooperation verlangten. $\mathrm{Zu}$ diesen Problemen rechnete er drohende Umweltkrisen, Unterentwicklung und Armut und nicht zuletzt die Gefahren des Wettrüstens. Mit dem schmählichen Untergang des großen Versuchs, der kapitalistischen Welt eine Alternative entgegenzusetzen, war das auf ideologischen Streit und militärische Überlegenheit fixierte Gegeneinander in der Weltpolitik am Ende angelangt. Der Weg schien frei, sich konstruktiv den gemeinsamen Überlebensfragen zuzuwenden.

Ein Fenster der Gelegenheit öffnete sich. Mit der Charta von Paris 1990 wurde eine Friedensordnung von Vancouver bis Wladiwostok skizziert, das Wort von der Friedensdividende machte die Runde. Mit den verschiedenen UNOGipfelkonferenzen zu Beginn dieses Jahrzehnts rückten Fragen einer gedeihlichen sozialen, wirtschaftlichen und ökologischen Weltentwicklung ins Zentrum des Interesses, wurden weitreichende Vereinbarungen zum Kampf gegen Hunger und Armut, gegen Umweltzerstörung und Unterdrückung erzielt. Der damalige UN-Generalsekretär Boutros-Ghali legte 1992 mit der Agenda für den Frieden ein umfassendes Konzept vor, wie die Vereinten Nationen ihre Schlüsselrolle bei der Entwicklung und Sicherung des Weltfriedens wahrnehmen sollten.

Es ist heute notwendig, sich dieser Prozesse zu erinnern. Dort wurden programmatische Grundlagen gelegt, auf denen aufgebaut werden kann: Sicherheit kann im globalen Maßstab nur noch gemeinsam, nicht mehr zu Lasten anderer erreicht werden; Frieden muss vor allem auf der Entwicklung einer sozial gerechteren, ökologisch bewussteren und demokratischer gestalteten Welt gründen; Stabilität kann nur durch verlässliche rechtliche Regularien erreicht werden - nicht durch militärische Überlegenheit.

\section{Globalisierung und Gewalt}

Tatsächlich brachten die neunziger Jahre eine weitreichende Zäsur. Doch die Weichen wurden in eine andere Richtung gestellt. Bestimmend für die globale Entwicklung war nicht Rio 1992, sondern Marrakesch 1994. Die Gründung der Welthandelsorganisation WTO symbolisierte, wie weit sich die Globalisierung unter den Vorzeichen neoliberaler Freihandelsdogmen institutionell verfestigt hatte. Bestimmend war nicht Paris 1990, sondern der Golfkrieg 1991 und die neue strategische Positionierung der NATO auf ihrem Gipfel von Rom (1991). Die »neue Weltordnung « des damaligen US-Präsidenten Bush sollte dem Worte nach zwar die Herrschaft des Rechts in den internationalen Beziehungen etablieren, beinhaltete aber im Kern das Recht militärischer Einmischungen durch die mit der »internationalen Gemeinschaft« identisch gesetzte westliche Staatenallianz. Das nach dem Ost-West-Gegensatz übriggebliebene Militärbündnis NATO gab sich die neue Aufgabe, ggf. weltweit Krisen »managen« zu wollen. Diese Entscheidung hatte unter anderem eine Konsequenz: Die Streitkräfte wurden auf Interventionsfähigkeiten »out of area« umgerüstet.

Der UNO wurden die nötigen Mittel zur Bewältigung der neuen Konflikte verweigert und somit erreicht, dass sie sich bei ihren Streitschlichtungsbemühungen von Mogadischu bis Srebrenica blamierte. Sie konnte anschließend an den Rand 